\title{
Celle des riches et celle des pauvres
}

Provenances, qualité et typicité de la maniguette d'Éthiopie

The one for the rich and the one for the poor: Provenances, quality and specificity of Ethiopian malagueta pepper

\section{Bernard Roussel et Feleke Woldeyes}

\section{OpenEdition \\ Journals}

Édition électronique

URL : http://journals.openedition.org/ethnoecologie/1288

DOI : 10.4000/ethnoecologie.1288

ISSN : 2267-2419

\section{Éditeur}

Laboratoire Eco-anthropologie et Ethnobiologie

\section{Référence électronique}

Bernard Roussel et Feleke Woldeyes, "Celle des riches et celle des pauvres », Revue d'ethnoécologie

[En ligne], 3 | 2013, mis en ligne le 12 novembre 2013, consulté le 19 avril 2019. URL : http:// journals.openedition.org/ethnoecologie/1288 ; DOI : 10.4000/ethnoecologie.1288

Ce document a été généré automatiquement le 19 avril 2019.

\section{(†) $९$}

Revue d'ethnoécologie est mis à disposition selon les termes de la licence Creative Commons Attribution - Pas d'Utilisation Commerciale - Pas de Modification 4.0 International. 


\title{
Celle des riches et celle des pauvres
}

\author{
Provenances, qualité et typicité de la maniguette d'Éthiopie \\ The one for the rich and the one for the poor: Provenances, quality and \\ specificity of Ethiopian malagueta pepper
}

Bernard Roussel et Feleke Woldeyes

1 L'Éthiopie est en passe de se doter d'un instrument juridique et institutionnel de protection des indications géographiques (Roussel \& Verdeaux 2007b). Piloté par une Agence d'État, chargée des questions de politiques environnementales et placée directement sous la responsabilité du Cabinet du Premier Ministre, l'Environmental Protection Authority, ce projet $^{1}$ affiche d'emblée une volonté claire: mettre la reconnaissance d'appellations d'origine au service de la protection des savoir-faire sur la nature et de la valorisation d'éléments de la biodiversitée, en l'occurrence celle des jardins d'Éthiopie. En liaison avec ce projet, des recherches multidisciplinaires ont été conduites $^{3}$ qui recensent les spécialités locales d'Éthiopie, fort nombreuses, analysent leurs conditions de production, de commercialisation et de consommation et décrivent leur place dans les circuits commerciaux, leur ancrage dans le local et leurs liens avec la diversité biologique et culturelle (Roussel, 2007). Parmi les produits très diversifiés (des cafés aux épices, des beurres aux miels, des viandes aux fruits) qui ont d'emblée retenu l'attention, figure la kororima (Boisvert et al. 2007). Épice très emblématique des cultures éthiopiennes, produite dans plusieurs régions d'Éthiopie où son potentiel économique a attiré les développeurs et les investisseurs, elle est vendue sur le marché national en référence à plusieurs provenances reconnues non pas tant pour leur originalité que pour la différence de qualité de leurs produits.

2 Cet article a pour objectif d'interroger les liens qui existent entre ces diverses provenances, leur typicité et la qualité de leurs produits dans la perspective des dynamiques de valorisation actuelles. On s'interrogera sur l'origine des caractéristiques des différentes provenances, sur la nature et l'élaboration des normes de qualité et sur leur évolution actuelle. Certaines des provenances concernées font l'objet de projets de développement locaux et de valorisation (Brunet \& Boisvert 2007 ; Feleke \& Roussel 2007). 
Réussissent-elles à trouver un marché ? Ne risque-t-on pas, en agissant sur la qualité, de perturber à terme les équilibres économiques actuels et la place des diverses provenances sur le marché national?

\section{De nombreux produits, de multiples provenances}

3 Au sein des végétaux et des productions végétales, les Amharas des hauts plateaux éthiopiens distinguent une catégorie qemäma qeman qui regroupe épices, aromates et plantes condimentaires (Chouvin 2000). C'est de là qu'est issue l'expression qemäma tera qui désigne sur tous les marchés d'Éthiopie, en région amhara comme ailleurs, l'endroit du marché, une ou plusieurs allées complètes ou simplement un groupe de quelques étalages, où sont présentées à la clientèle, ces produits d'usages quotidiens, indispensables à la cuisine de tous les jours. Parmi eux figure, toujours en bonne place, la kororima.

4 On l'y trouve sous diverses formes (Figure 1). Les fruits secs entiers sont les plus fréquents ; les consommateurs les préfèrent ainsi car la paroi déshydratée du péricarpe, très résistante après dessiccation, protège son contenu de graines et lui conserve son pouvoir aromatique. Cependant, dans les villes surtout, destinées à une clientèle urbaine "plus pressée ", on trouve de plus en plus souvent des graines et même des poudres: selon les besoins journaliers, le décorticage est généralement fait par les détaillants finaux sur les marchés d'Addis-Abeba ou dans les petits souks locaux, pour ajouter un peu de valeur au produit. Quant à la poudre de graine, elle est conditionnée dans des sacs en plastique ou des pots par des petites unités de transformations spécialisées dans les préparations alimentaires domestiques, les balten $a^{4}$ : c'est essentiellement sous cette forme que la kororima est présente dans les supermarchés et les boutiques. Elle y est rarement en poudre pure mais souvent dans des mélanges pluri-épices tels le shiro, l'awase ou le fameux deleh-berbere, préparation condimentaire très appréciée et emblématique de la cuisine nationale éthiopienne contemporaine (Tableau 1).

5 Le plus souvent, et même dans les grandes cités modernes, le décorticage des kororimas est une tâche avant tout ménagère et les maîtresses des maisons respectables préparent elles-mêmes leur deleh-berbere ${ }^{5}$, choisissant soigneusement les composants, les grillant avec soin et les portant elles-mêmes au moulin public. Les recettes sont des sortes de patrimoines familiaux transmis de génération en génération par les femmes âgées.

6 Actuellement, le marché international de la kororima n'est guère florissant et ne représente qu'une faible part de la production de toute l'Éthiopie: en moyenne, sur les 5625 tonnes produites annuellement, seulement 255 tonnes sont exportées (soit moins de $5 \%$ selon Brunet \& Boisvert 2007) car elle pâtit de la concurrence avec les vraies cardamomes asiatiques (la cardamome verte, Elettaria cardamomum White \& Masson; et la cardamome noire, Amomun subulatum Roxb.) avec lesquelles elle se trouve d'ailleurs souvent amalgamée dans les statistiques commerciales. Il faut dire que toutes ces épices sont très proches sur le plan des usages et des propriétés: ainsi l'huile essentielle de kororima a quasiment la même composition que les huiles de cardamomes et n'arrive pas à conquérir une place spécifique sur les marchés internationaux pourtant très demandeurs (Feleke \& Roussel 2008).

7 Du point de vue botanique, le fruit est une capsule bacciforme rouge à maturité qui n'est que rarement vendue fraîche et uniquement sur les marchés locaux: les paysans, et 
parfois les premiers collecteurs assurent eux-mêmes la dessiccation et, nous le verrons, les méthodes varient selon les provenances. Ce fruit est produit par une plante pérenne de la famille des Zingibéracées: Aframomum corrorima ${ }^{6}$ (Braun) Jansen, endémique d'Éthiopie qui pousse spontanément dans les sous-bois un peu clairs, sous ombre légère ou dans les bas-fonds humides entre $1500 \mathrm{~m}$ et $2000 \mathrm{~m}$ d'altitude dans des conditions écologiques proches de celles du café arabica: si son aire d'origine correspond probablement à celle du café spontané (c'est-à-dire le massif montagnard forestier du sud-ouest de l'Éthiopie appelée la Coffee Forest (Jansen 1981; Goettsch 1991), sa distribution actuelle est bien plus étendue car elle est maintenant cultivée dans les nombreux jardins de toute la zone sud de l'Éthiopie.

Bien qu'à notre connaissance, aucune étude génétique précise n'ait porté sur la kororima, aucune variation infraspécifique n'a été rapportée: nos observations de terrains et les réponses de nos informateurs paysans aux questions concernant l'existence éventuelle de cultivars ont toujours été négatives. Transplantée d'une région à l'autre, la kororima adopte les caractères de celle de sa région d'adoption; des kororimas spontanées mises en culture ne se distinguent pas de leurs voisines cultivées depuis longtemps. C'est pourquoi les acteurs de la filière, des cultivateurs aux marchands s'accordent tous pour affirmer que les différences observées entre les maniguettes d'origine différentes sont dues aux conditions de sols et de climat, aux pratiques culturales et surtout à la manière dont les fruits sont cueillis, nettoyés et séchés c'est-à-dire aux savoir-faire des producteurs.

Figure 1. Kororima, fruits secs et décortiqués, graines et poudre

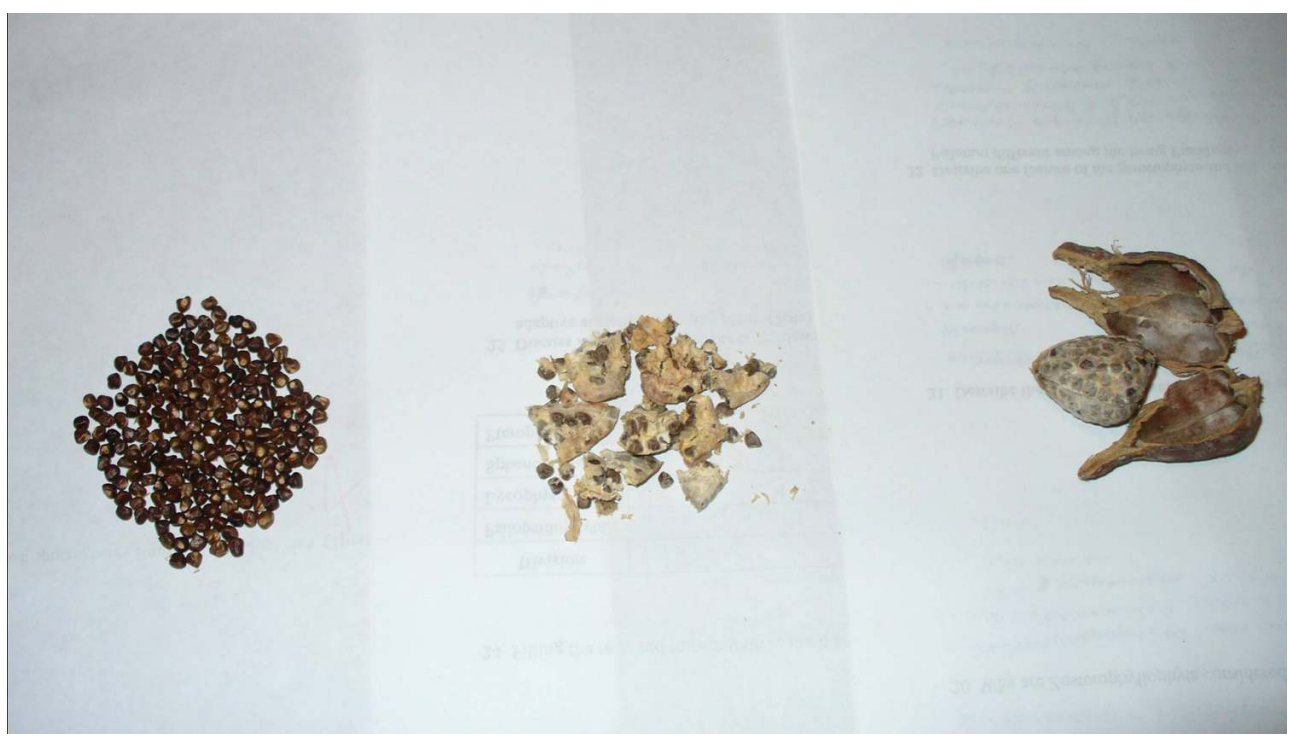

Photos F.W.

Tableau 1. Quelques préparations culinaires courantes utilisant la kororima 


\begin{tabular}{|c|c|}
\hline - qey/alicha wot & - ragoût pimenté ou non (rouge/jaune) de viande ou de légumineuses \\
\hline - kitfo & - viande hachée, crue ou à peine cuite \\
\hline - dulet & - tripes de moutons ou de boeuf \\
\hline$-a y b$ & - fromage blanc assaisonné accompagnant les ragoûts ou la viande haché \\
\hline - ye-inqulal ferfer/tibis & - oeufs brouillés/omelette \\
\hline - besso & - bouillie plus ou moins liquide de farine d'orge grillée \\
\hline - chikko & - farine d'orge grillée délayée dans du beurre fondu \\
\hline - dabo-qolo & - petits biscuits secs constituées de farine de blé grillés ou frits \\
\hline - awaze & $\begin{array}{l}\text { - pâte condimentaire plus ou moins liquide obtenue par dilution dans un alcool local d'une poudre de piment pure ou de } \\
\text { deleh-berbere }\end{array}$ \\
\hline - deleh-berbere & $\begin{array}{l}\text { - poudre condimentaire à base de piment fort et de nombreux autres épices et aromates : poivre, gingembre, nigelle, } \\
\text { ajowan, ail, échalotes..., }\end{array}$ \\
\hline - shiro & - farine ou bouillie de pois ou de pois chiche \\
\hline - yetenetere qibe & - beurre épicé \\
\hline - daatta/qocqoca & $\begin{array}{l}\text { - pâte condimentaire plus ou moins liquide et contenant des petits piments mitmita et de nombreux autres condiments et } \\
\text { aromates }\end{array}$ \\
\hline - ye-sinde dabbo & - pain brun de blé entier \\
\hline - ye-furno dabbo & - pain blanc de blé \\
\hline - bunna & - décoction de café \\
\hline - shameta & - boisson fermentée à base de farine de blé diluée \\
\hline
\end{tabular}

Figure 2. Fruits mûrs de kororima montrant l'endocarpe charnu, blanc et juteux où sont logées les graines brunes

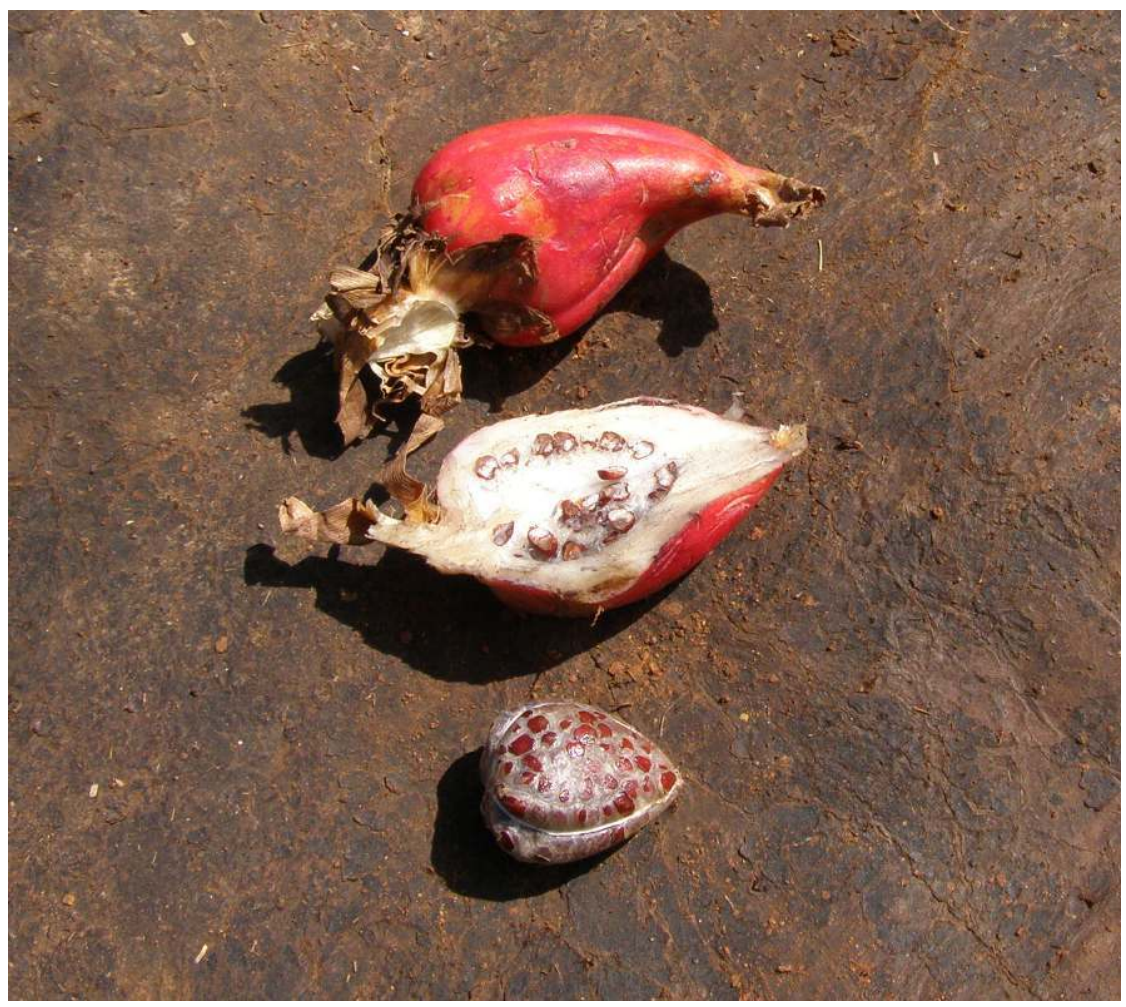

Photos B.R

9 Sur les marchés de la capitale, lorsque l'on relève l'origine des kororimas vendues, deux provenances émergent majoritairement et sont généralement présentes conjointement sur tous les étalages ${ }^{7}$. En fait si ces deux noms cachent des origines multiples comme le montrent les tableaux 2 et 3 , ils correspondent à deux filières principales, géographiquement bien disjointes, qui ne peuvent donc se mélanger avant leur arrivée chez les marchands de la capitale. L'une est issue de l'ensemble de la zone de la Coffee Forest et ses produits prennent le nom de l'ancienne province impériale du Kafa : ye-Kafa kororima (les kororimas du Kafa). L'autre, vendue sous le nom de ye-Gofa kororima, 
rassemble tout un ensemble de provenances de la région d'Arba Minch, qui était autrefois la capitale de la Province du Gamo-Gofa. Chez les grossistes et les détaillants d'AddisAbeba, les kororimas sont encore dans des sacs bien distincts ${ }^{8}$ et arrivent, dans des camions séparés, des centres régionaux bien distincts et distants géographiquement (cf. Carte 1) : il n'y a pas de mélange entre les deux grandes provenances. En aval de la capitale, en particulier pour les kororimas qui sont envoyées sur les marchés du Nord du pays, les deux provenances essentielles se fondent et se mélangent sous le nom de « kororima d'Addis ». Quant au marché international, il ne se préoccupe pas de l'origine géographique des produits et la dénomination la plus localisée ne fait référence qu'à l'Éthiopie dans son ensemble (par exemple « fausse cardamome d'Éthiopie » Tableau 2).

Au sein de chacune des deux grandes filières, le mélange des provenances commence très tôt dans les chaînes commerciales: ainsi dès le marché de concentration de Laska (Tableau 2) au cœur de l'aire de production de la kororima de Basketo, il y a réunion des diverses petites provenances locales, à raison de trois kororimas de Basketo pour une provenant d'ailleurs. Les causes en sont tout d'abord l'absence de voies de communication carrossables: les petites provenances mal ou pas du tout reliées aux voies routières principales fusionnent avec celles qui bénéficient d'une bonne desserte (Feleke \& Roussel, 2008). En outre, ces mélanges permettent de palier les manques lorsque la production locale principale du marché de concentration est un peu déficitaire.

Plus loin dans la filière, au niveau de chacun des principaux marchés zonaux (Bulki et Sawla, Soddo et Arba Minch), de nouvelles provenances viennent s'amalgamer. C'est au niveau des marchés régionaux (Shashamane et Awassa) qui jouent le rôle de centres pour toutes ces provenances de kororimas, qu'elles finissent par se confondre dans une même appellation : ye-Gofa kororima.

Tableau 2. Provenances et évolution du nom le long de la filière de la kororima du Gofa

\begin{tabular}{|c|c|c|c|c|c|c|c|c|c|}
\hline 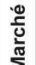 & Laska & \multicolumn{2}{|c|}{ Bulki \& Sawla } & $\begin{array}{l}\text { Soddo \& } \\
\text { AlMinch }\end{array}$ & $\begin{array}{c}\text { Shashemene } \\
\text { \& Awassa }\end{array}$ & Addis Ababa & \multicolumn{2}{|c|}{ National } & International \\
\hline \multirow{7}{*}{ 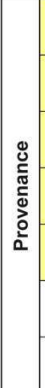 } & $\begin{array}{l}\text { Ye-Basketo } \\
\text { kororima }\end{array}$ & $\begin{array}{l}\text { Ye-Basketo } \\
\text { kororima }\end{array}$ & \multirow{3}{*}{$\begin{array}{c}\text { Ye-Basketo } \\
\text { kororima }\end{array}$} & \multirow{4}{*}{$\begin{array}{l}\text { Ye-Gofa } \\
\text { kororima }\end{array}$} & \multirow{5}{*}{$\begin{array}{l}\text { Ye-Gofa } \\
\text { kororima }\end{array}$} & \multirow{5}{*}{$\begin{array}{l}\text { Ye-Gofa } \\
\text { kororima }\end{array}$} & \multirow{5}{*}{$\begin{array}{l}\text { Ye-Gofa } \\
\text { kororima }\end{array}$} & \multirow{7}{*}{$\begin{array}{l}\text { Ye-Addis } \\
\text { kororima }\end{array}$} & kororima \\
\hline & $\begin{array}{l}\text { Ye-Malo } \\
\text { kororima }\end{array}$ & $\begin{array}{l}\text { Ye-Malo } \\
\text { kororima }\end{array}$ & & & & & & & $\begin{array}{l}\text { cardamome } \\
\text { korarima }\end{array}$ \\
\hline & $\begin{array}{l}\text { Ye-Gelila } \\
\text { kororima }\end{array}$ & $\begin{array}{l}\text { Ye-Gelila } \\
\text { kororima }\end{array}$ & & & & & & & $\begin{array}{l}\text { cardamome } \\
\text { muscade }\end{array}$ \\
\hline & & \multicolumn{2}{|c|}{$\begin{array}{l}\text { Ye-Gazer } \\
\text { kororima }\end{array}$} & & & & & & \\
\hline & & & & $\begin{array}{l}\text { Ye-Kamba } \\
\text { Kororima }\end{array}$ & & & & & cardamome \\
\hline & & & & & & $\begin{array}{l}\text { Ye-Bonga-Kafa } \\
\text { kororima }\end{array}$ & Ye-Kafa & & $\begin{array}{l}\text { cardamome } \\
\text { éthiopienne }\end{array}$ \\
\hline & & & & & & $\begin{array}{l}\text { Ye-Bench-Maji } \\
\text { kororima. }\end{array}$ & & & $\begin{array}{l}\text { maniguette } \\
\text { éthiopienne }\end{array}$ \\
\hline
\end{tabular}

12 La situation est similaire pour les provenances de la Coffee Forest (Tableau 3) : au niveau zonal (Bonga), il y a fusion de plusieurs petites provenances mais au niveau de Jimma (le marché régional) : deux origines continuent à être distinguées et vendues séparément (Bench-Maji et Bonga). Ce n'est qu'à leur arrivée à Addis-Abeba où elles figurent parfois encore séparément, et lorsqu'elle partent sur le marché national, qu'elles sont à leur tour confondues dans un même appellation qui peut être Ye-Jimma kororima mais qui le plus souvent est présentée par les vendeurs sous le nom de Ye-Kafa kororima. simplification de la nomenclature des provenances sur les marchés de la Capitale. Si la 
plupart des provenances restent présentes, le public n'en sait généralement rien et la grande majorité des commerçants ne présentent à la vente que deux "grands noms ". Interrogés sur la raison d'une telle simplification, les vendeurs sont unanimes: contrairement aux clients plus ruraux des zones de productions, les consommateurs, mais aussi les détaillants des centres urbains et des régions éloignées des aires d'origine, ignorent l'existence de la plupart des provenances, ou, s'ils en ont entendu parler ne connaissent pas leurs spécificités. Ils ne sont généralement pas à la recherche d'une origine, avec une spécificité particulière, mais se préoccupent avant tout de la qualité des produits et de leur prix. Pourtant, les multiples provenances présentes sur le marché éthiopien offrent tout un éventail de particularités originales qui s'accompagnent aussi de différences dans la qualité évaluée selon des critères complexes par les divers acteurs de la filière.

Tableau 3. Provenances et évolution du nom le long de la filière de la kororima du Kafa

\begin{tabular}{|c|c|c|c|c|c|c|c|}
\hline 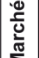 & Bonga & Jimma & \multicolumn{2}{|c|}{ Addis Ababa } & \multicolumn{2}{|c|}{ National } & International \\
\hline \multirow{5}{*}{ 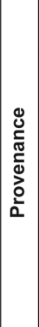 } & $\begin{array}{l}\text { Ye-Gaballa/ } \\
\text { Telo kororima }\end{array}$ & $\begin{array}{l}\text { Ye-Bonga } \\
\text { kororima }\end{array}$ & $\begin{array}{c}\text { Ye-Bonga-Kafa } \\
\text { kororima }\end{array}$ & \multirow{4}{*}{$\begin{array}{l}\text { Ye-Jimma } \\
\text { kororima }\end{array}$} & \multirow{4}{*}{$\begin{array}{l}\text { Ye-Kafa } \\
\text { kororima }\end{array}$} & \multirow{5}{*}{$\begin{array}{l}\text { Ye-Addis } \\
\text { kororima }\end{array}$} & kororima \\
\hline & $\begin{array}{l}\text { Ye-Decha } \\
\text { kororima }\end{array}$ & & & & & & $\begin{array}{c}\text { cardamome } \\
\text { korarima }\end{array}$ \\
\hline & $\begin{array}{l}\text { Ye-Gimbo } \\
\text { kororima }\end{array}$ & & & & & & $\begin{array}{l}\text { cardamome } \\
\text { muscade }\end{array}$ \\
\hline & & $\begin{array}{l}\text { Ye-Benj-Maji } \\
\text { kororima }\end{array}$ & $\begin{array}{l}\text { Ye-Bench-Maji } \\
\text { kororima }\end{array}$ & & & & $\begin{array}{c}\text { fausse } \\
\text { cardamome }\end{array}$ \\
\hline & & & \multicolumn{2}{|c|}{$\begin{array}{l}\text { Ye-Gofa } \\
\text { kororima }\end{array}$} & $\begin{array}{l}\text { Ye-Gofa } \\
\text { kororima }\end{array}$ & & $\begin{array}{l}\text { cardamome } \\
\text { éthiopienne } \\
\text { maniguette } \\
\text { éthiopienne }\end{array}$ \\
\hline
\end{tabular}

\section{Des provenances aux caractères bien marqués} leurs propres kororimas de celles des provenances voisines : ainsi en pays Basketo, les fermiers peuvent décrire les particularités de leur kororima par rapport à celle qui provient des petites zones de productions environnantes (Ye-Malo kororima, Ye-Gelila kororima, Ye-Dime kororima) et la tiennent, de plus, comme de bien meilleure qualité. Ils insistent en particulier sur la couleur des fruits frais (d'un rouge brillant à maturité) et sur la forme globuleuse sub-sphérique du fruit sec. Ce dernier caractère est pour les fermiers et les commerçants locaux un signe distinctif essentiel mais aussi un critère de qualité très apprécié. Il n'est pas lié par les fermiers avec une quelconque spécificité " génétique " " puisqu'un pied de maniguette prélevé à Malo, Gelila ou ailleurs et planté en Basketo finit toujours par produire des fruits bien sphériques, nous disent-ils, sous l'influence du milieu local et des pratiques culturales. Mais la forme sphérique est avant tout due à la manière de nettoyer les fruits avant de les mettre à sécher : d'un coup de lame habile, les restes secs du périanthe, au sommet des fruits, du pédoncule floral et des bractées à la base, sont enlevés avant que les maniguettes ne soient étalées au soleil sur des claies de bambous : les fermiers disent qu'elles sont « circoncises » (tegereze).

Ce sont les marchands, et tout particulièrement les grossistes (jimla negade) au niveau des centres régionaux et nationaux, qui ont la vision globale la plus complète concernant l'originalité des nombreuses provenances de la kororima éthiopienne. Les critères retenus (Tableau 4) concernent le fruit entier (sa forme, sa couleur, sa fermeté, son 
poids...) mais aussi les graines (leur nombre, leur couleur, leur poids, leur odeur). Après décorticage, il n'est pas toujours facile de reconnaitre l'origine de la kororima : certains détaillants sont cependant capables de faire une différence entre les graines des deux grandes provenances. Les particularités les plus faciles à reconnaître sont celles qui sont liées aux techniques de séchage et qui diffèrent selon les zones de production : ainsi, dans les climats très humides de la Coffee Forest, les maniguettes du Kafa sont enfilées sur une corde (en fibre de faux bananier ensète) et mises à sécher au-dessus des foyers familiaux : elles apparaissent donc percées et leur couleur est toujours noirâtre à cause de la fumée ${ }^{10}$.

Figure 3. Fruits secs de kororima du Kafa montrant le trou d'enfilage de la corde en fibres d'ensète utilisée pour sa dessiccation et son fumage

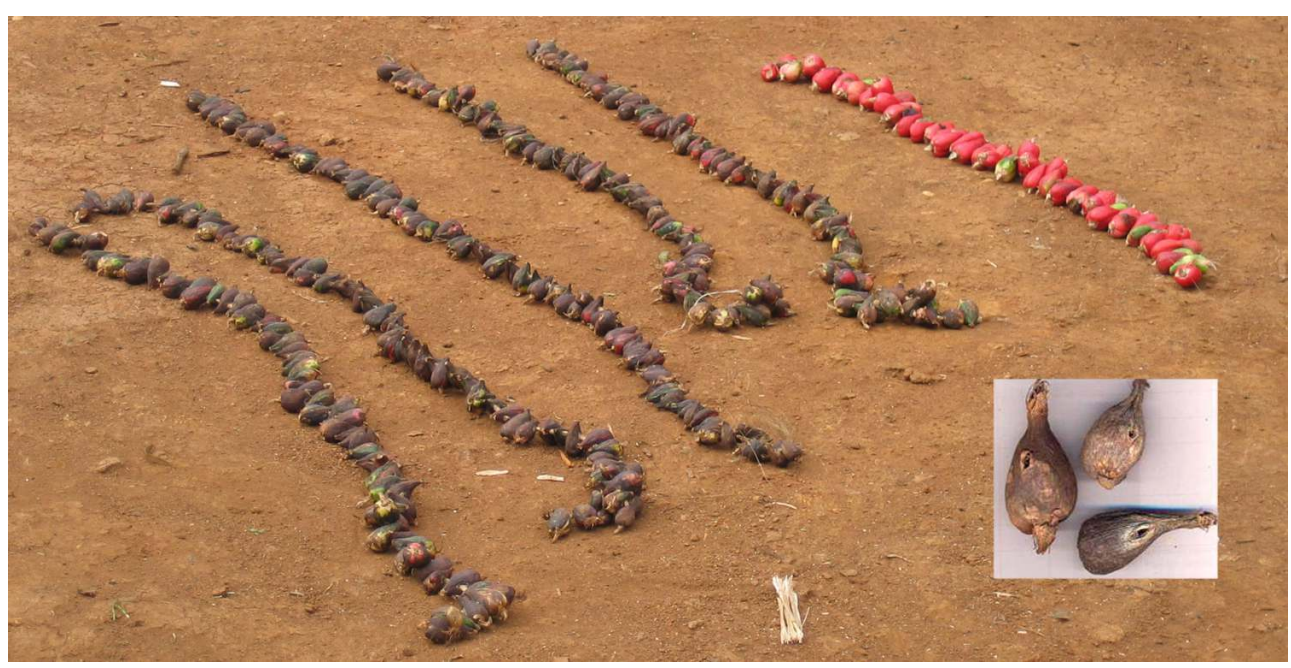

Beaucoup des particularités retenues sont également des critères de qualité. Par exemple, la taille du fruit, celle des graines est un bon indicateur du fait que la cueillette a eu lieu au moment exact de la maturité et constitue une garantie de saveur. Pour les commerçants, la fermeté est un caractère distinctif important: les fruits doivent être suffisamment fermes (tenkara) ce qui traduit une bonne maîtrise du processus de dessiccation et est une garantie contre les attaques de moisissure et contre les pertes d'eau importantes (donc de poids et de valeur, puisque les kororimas sont vendues au poids) lors du transport. Mais cette fermeté ne doit pas être excessive au risque de rendre le décorticage laborieux voire impossible. Les commerçants apprécient tout particulièrement les provenances dont les kororimas ont des enveloppes robustes ce qui fait qu'elles n'explosent pas dans les sacs de transports et que les graines sont protégées et gardent saveur et odeur. Mais il ne faut pas qu'elles soient trop lourdes ou épaisses ce qui rendraient l'égrenage peu rentable. 
Tableau 4. Caractères distinctifs des deux grandes catégories de kororimas vues par les commerçants

\begin{tabular}{|c|c|c|c|}
\hline Nature du produit & Critères & Kororima du Gofa & Kororima du Kafa \\
\hline \multirow{4}{*}{ Fruit entier sec } & Aspect (couleur et appendices) & neta-yale (brun-pale) & $\begin{array}{l}\text { tikur wozam (noir brillant). Présence d'un } \\
\text { trou au sommet du fruit. Odeur de fumée. }\end{array}$ \\
\hline & Taille & $\begin{array}{l}\text { ye tegerase : "circoncise", sphérique } \\
\text { avec une légère dépression à la base }\end{array}$ & reste important des appendices \\
\hline & Fermeté & tenkara (ferme) & $\begin{array}{l}\text { paroi du fruit résistante mais facile à } \\
\text { décortiquer pour extraire les graines. }\end{array}$ \\
\hline & Poids & fruits sphériques gros et lourds & fruits petits et allongés \\
\hline \multirow{3}{*}{ Graines } & Quantité & graines nombreuses et polyédriques & graines peu nombreuses et sphériques \\
\hline & Taille et couleur & brunes et brillantes & noires et ternes \\
\hline & Arôme & arôme (shita) fort & arôme doux \\
\hline
\end{tabular}

Il est difficile de caractériser sur le plan statistique la différence entre les maniguettes du Kafa et celles de Bonga vendues sur le plus grand marché d'Addis-Abeba (le Mercato). Le tableau 5 réunit des mesures faites sur quatre sortes de kororimas : ye-Basketo kororima viennent directement du marché de Laska et ne sont donc qu'un mélange de quelques provenances voisines. Ye-Gofa kororima sont un mélange de plusieurs provenances non identifiées mais toutes issues du Gamo-Gofa. Ye Bonga kororima nous a été donné comme appartenant à une provenance pure et Ye-Kafa kororima comme un mélange de plusieurs origines inconnues.

Tableau 5 - Analyse quantitative de plusieurs provenances du Mercato d'Addis-Abeba

\begin{tabular}{|c|c|c|c|c|}
\hline & Ye-Basketo kororima & Ye-Gofa kororima & Ye-Bonga kororima & Ye-Kafa kororima \\
\hline Nombre de fruits par $\mathrm{kg}$ & $162( \pm 12)$ & $150(+17)$ & $180(+50)$ & $168(+34)$ \\
\hline Poids des fruits (g) & $6.01( \pm 0,81)$ & $6.71(+0,, 92)$ & $5,97(+1,71)$ & $6,13(+1,01)$ \\
\hline Longueur du fruit $(\mathrm{cm})$ & $4.6(+0,17)$ & $4,7(+0,21)$ & $6,30(+0,66)$ & $5,52(+0,51)$ \\
\hline Circonférence (cm) & $7.66( \pm 0,55)$ & $7,3(+0,68)$ & $7,13(+0,49)$ & $7,02(+0,68)$ \\
\hline Rapport longueur circonférence & $0.5995(+0,04)$ & $0,634(+0,051)$ & $0,886(+0,10)$ & $0,791(+0,099)$ \\
\hline $\begin{array}{l}\text { Pourcentage du poids par Rapport aux graines de } \\
\text { fruit mur par gousse }\end{array}$ & $\begin{array}{l}74.45 \% \\
25.55 \%\end{array}$ & $\begin{array}{l}65,88 \\
34,12\end{array}$ & $\begin{array}{l}56,03 \\
43,97\end{array}$ & $\begin{array}{l}63,78 \\
36,22\end{array}$ \\
\hline Nombre total de graines par gousse & $198(+9)$ & $198(+11)$ & $192(+78)$ & $213(+46)$ \\
\hline Le poids moyen des graines $(\mathrm{g}$ ) & 0.024 & $0,022(+0.003)$ & $0,018(+0,006)$ & $0,018(+0,003)$ \\
\hline
\end{tabular}

Des résultats contenus dans ce tableau, on retiendra surtout des différences au niveau de l'homogénéité. Au sein de chacune des provenances, les caractères les plus stables concernent la forme des fruits (sphérique du coté de Gofa, plus allongée dans le Kafa), le poids et surtout le nombre de graines (plus élevés dans le cas des maniguettes du Gofa). D'une manière globale, il apparaît assez nettement que les kororimas du Gofa forment un ensemble plus homogène : peut-être doit-on y voir un reflet des conditions et pratiques de production? En effet, les kororimas du Gofa sont toutes cultivées, ce qui permet à la fois un meilleur contrôle de la croissance et de la maturation, alors que celles du Kafa sont encore pour une grande part issues de la cueillette en forêt (même si une large proportion d'entre elles provient toujours, et de plus en plus, des jardins.

D'une manière globale aussi, ces résultats montrent très clairement que les différences entre ces deux ensembles de provenances peuvent au-delà de quelques spécificités 
d'aspect (la couleur, la forme) être hiérarchisés en termes de qualité. Finalement, les kororimas du Kafa présentent, nous allons le voir, un déficit de qualité sur l'ensemble de leurs caractéristiques. Sur la plupart des étalages de la capitale, le niveau de qualité est présenté comme lié à l'origine : le plus élevé est toujours attribué à la kororima du Gofa alors que celle du Kafa est toujours considérée comme de qualité moindre. Cette situation est d'ailleurs admise par tous les acteurs de la filière, des producteurs aux détaillants et aux consommateurs et se traduit, nous allons le voir, par une différence significative dans les prix.

\section{Deux grandes provenances aux qualités différentes}

Il n'y a pas encore d'instrument officiel assurant tout le long des filières de kororima la traçabilité des produits et le contrôle de leur qualité. Il existe cependant, dans le cadre du ministère de l'agriculture, des services de contrôle de la qualité ${ }^{11}$. Mais ils sont encore peu présents sur le terrain et le fonctionnement des filières repose sur les contacts et la confiance entre les divers niveaux des producteurs aux consommateurs: tableau 5. Chacun des maillons possède et contrôle ses propres critères et signes de qualité.

Ainsi les cultivateurs accordent une valeur importante à la couleur du fruit et à sa taille : ils sont des indicateurs de l'état de maturité qui conditionne à leurs yeux la qualité finale du produit. Les marchands sont particulièrement attentifs à la forme et à la fermeté des fruits secs et à l'intégrité et la résistance de la paroi, autant de signes qui permettent d'apprécier la qualité du séchage dont dépend in fine l'arôme de l'épice. Pour les consommateurs, la couleur des graines, leur taille et leur parfum sont autant de critères : une grosse taille, une couleur brune et brillante, une odeur forte sont considérées comme des signes de grande qualité.

Tableau 6. Importance (de 0 à 5+) accordée aux différents critères de qualité par les divers acteurs de la filière

\begin{tabular}{|c|c|c|c|c|}
\hline Nature du produit & Critères de qualité & Producteurs & Collecteurs/ marchands & Consommateurs \\
\hline \multirow{4}{*}{ Fruit frais } & Taille du fruit & +++++ & +++++ & \\
\cline { 2 - 5 } & Couleur (maturité) & ++++++ & +++ & \\
\hline \multirow{4}{*}{ Fruit sec } & Color et forme (appendices) & $+{ }^{+++}$ & +++++ & ++ \\
\cline { 2 - 5 } & Taille & +++++ & +++++ & +++++ \\
\cline { 2 - 5 } & Fermeté & +++ & +++++ & +++ \\
\cline { 2 - 5 } & Poids & +++++ & +++++ & ++++ \\
\hline \multirow{3}{*}{ Graine } & Quantité & +++ & +++++ & ++++ \\
\cline { 2 - 5 } & Taille & & +++++ & +++++ \\
\cline { 2 - 5 } & Arome & & & +++++ \\
\hline \multirow{2}{*}{ Poudre } & Arôme & & +++ \\
\hline
\end{tabular}

Sur les étalages les clients choisissent soigneusement les fruits secs, un par un et selon leurs critères personnels de qualité, ce qui amène souvent les détaillants à n'offrir à la vente qu'un choix réduit de kororimas (pour éviter les possibles comparaisons) et à faire des efforts de sélection et d'homogénéisation de leurs offres par élimination des fruits de mauvaise qualité (qui peuvent alors être valorisés après décorticage sous forme de graines : Brunet \& Boisvert, 2007, p. 38). La recherche d'une qualité élevée paraît bien être 
le critère important pour lequel les consommateurs sont prêts à payer plus cher. Le prix d'un kilogramme de graines de kororima du Gofa peut atteindre actuellement 50 birrs $^{12}$ à Addis-Abeba (120 birrs pour une kilogramme de poudre) alors que des quantités équivalentes de kororimas du Kafa peinent à trouver acheteurs pour 30 birrs. Tout le long de la chaîne, le différentiel de prix lié à la qualité - au sein d'une même provenance, jusqu'à trois niveaux différents de qualité sont distingués - n'est pas toujours aussi fort : de la ferme jusqu'aux étalages de détail, il est plutôt de l'ordre de 2 à 5 birrs par kilogramme de fruits secs.

En revanche, l'origine des produits ne semble pas être une préoccupation : la question de la provenance n'intervient que dans la mesure où elle parait associée à celle de la qualité. Le tableau 7 présente l'évaluation comparée des défauts de qualité des deux principales provenances de kororima par les marchands du Mercato d'Addis-Abeba. On voit bien qu'à leurs yeux, la kororima du Gofa est toujours de meilleure qualité quel que soit le critère envisagé. Et c'est cette représentation un peu simpliste que l'on trouve au niveau de leurs clients : toutes les kororimas du Gofa ne sont pourtant pas de bonne qualité ; les deux catégories sont des mélanges de provenances diverses.

Tableau 7. Défauts de qualité (de 0 à 3 ) et causes pour les deux provenances Gofa et Kafa

\begin{tabular}{|c|c|c|c|c|c|c|}
\hline \multirow[t]{2}{*}{ Problèmes } & \multicolumn{2}{|c|}{ Nature de la ressource } & \multicolumn{2}{|c|}{ Culture et transformation } & \multicolumn{2}{|c|}{ Conditions de transport et de stockage } \\
\hline & Gofa & Kafa & Gofa & Kafa & Gofa & Kafa \\
\hline Humidité résiduelle (ertebet) & & & 2 & 3 & 2 & 2 \\
\hline Moisissure (shagata) & & & 1 & 3 & 2 & 2 \\
\hline Perte de poids & & & & 3 & & 3 \\
\hline Déchets & & 2 & & 3 & & \\
\hline Hétérogénéité des fruits & 1 & 3 & & 2 & & \\
\hline Petite taille du fruit & 1 & 3 & & & & \\
\hline Fermeté faible du fruit & & & & 3 & & 2 \\
\hline Fruit vide de graines & & & & 3 & & 1 \\
\hline Petites graines & 1 & 3 & & 3 & & \\
\hline Aroma faible & 1 & 1 & & 3 & 1 & 3 \\
\hline Couleur terne des graines & & 3 & & 3 & & \\
\hline
\end{tabular}

Pour finir, il faut remarquer que, sur la plupart des étalages, les deux provenances, celle de basse qualité à un prix moindre et celle de qualité supérieure à un prix plus élevé, se côtoient. Il existe même des petits détaillants qui sont spécialisés dans la commercialisation de la kororima du Kafa. Cela suggère qu'il existe donc un marché double avec un produit cher et un autre peu coûteux. Le premier, la kororima du Gofa, est destiné à une clientèle riche ou qui le réserve à des utilisations prestigieuses, repas de fête ou confection du fameux condiment deleh berbere. Le second, la maniguette du Kafa, trouve sa clientèle parmi les personnes moins fortunées mais est souvent acheté par les industriels des baltena qui fabriquent des poudres et des mélanges tout prêts et que rien n'oblige à indiquer sur leurs sachets l'origine des produits.

Toutefois, la situation que nous venons de décrire est en train d'évoluer et l'on assiste à certains changements liés aux tentatives récentes de valorisation de la production éthiopienne de kororima. 


\section{Les tendances actuelles: des projets de développement qui changent l'équilibre commercial ancien}

Au cours de la dernière décennie, le gouvernement de la Région Sud de l'Éthiopie (SNNPR, The Southern Nations, Nationalities, and Peoples' Region) a attribué à la partie sud-ouest de son territoire un statut particulier de «zone de spécialisation» pour la production d'épices: des opérations gouvernementales ont été conduites (introductions de plants, formations,..) pour inciter les fermiers à améliorer et accroître leur production d'épices : kororima, poivre long (timiz), piment long (berbere), gingembre mais aussi des introductions nouvelles (poivre noir, vraie cardamome).

Dans la région du Gofa, autour d'Arba Minch, les actions en faveur de la production de kororima ont surtout consisté en la diffusion de plants pour la culture dans les jardins : certaines provenances ont ainsi progressé et occupent maintenant une place de plus en plus importante sur les marchés : c'est le cas par exemple de la maniguette produite dans les jardins de Kamba qui devient, sur les marchés régionaux, la principale rivale de celle de Basketo. Cette dernière provenance a été choisie par le "Projet Jardin d'Éthiopie » (Boisvert et al., 2007) car elle est entièrement produite dans de remarquables jardins domestiques, à l'organisation originale en lanière et à la biodiversité élevée (cf. Figure 4) qui doivent, pour perdurer, être mieux valorisés sur le plan économique. L'objectif à court terme est de mettre en place une appellation d'origine sur cette provenance qui constitue une part essentielle de la ye-Gofa kororima : les études nécessaires à la rédaction du cahier des charges sont en cours (Feleke \& Roussel, 2008).

Figure 4. Jardin en lanière du pays basketo

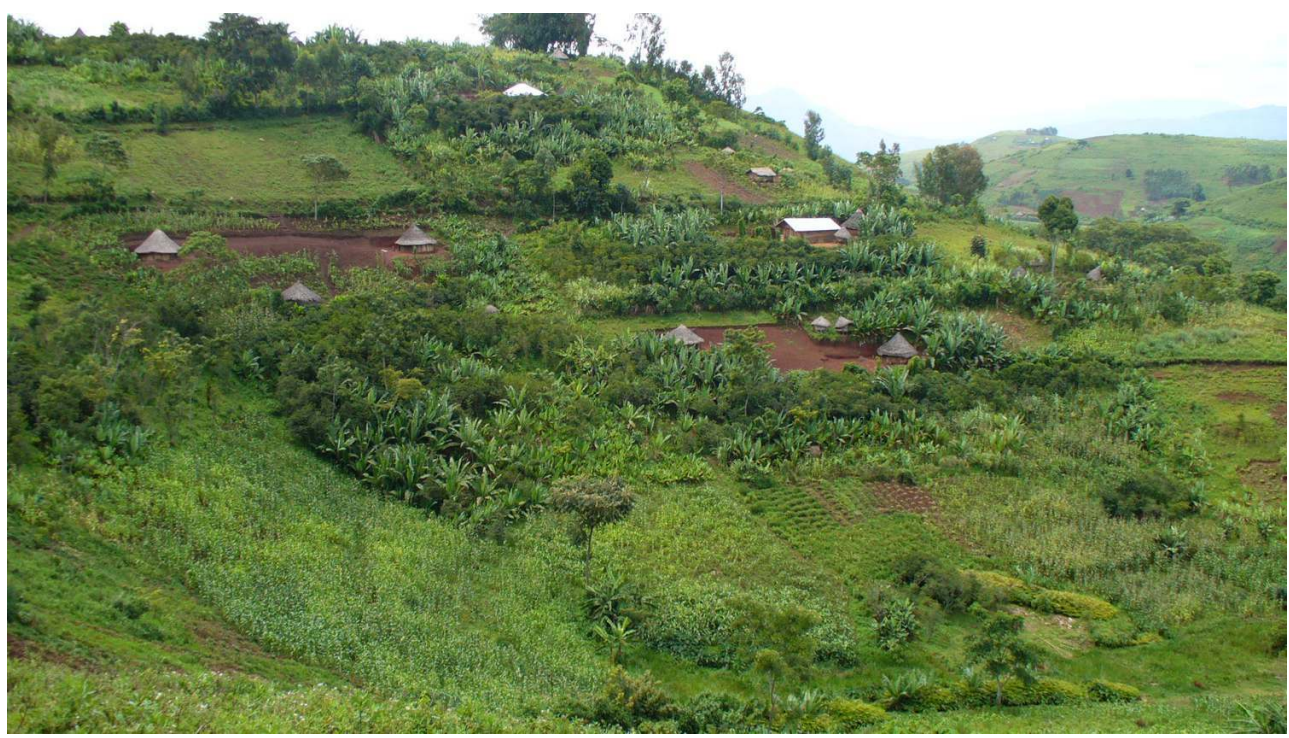

Les kororimas sont produites dans les parties basses et humides des jardins ou, à mi-pente, à l'ombre des grands arbres

Photo B. R. 


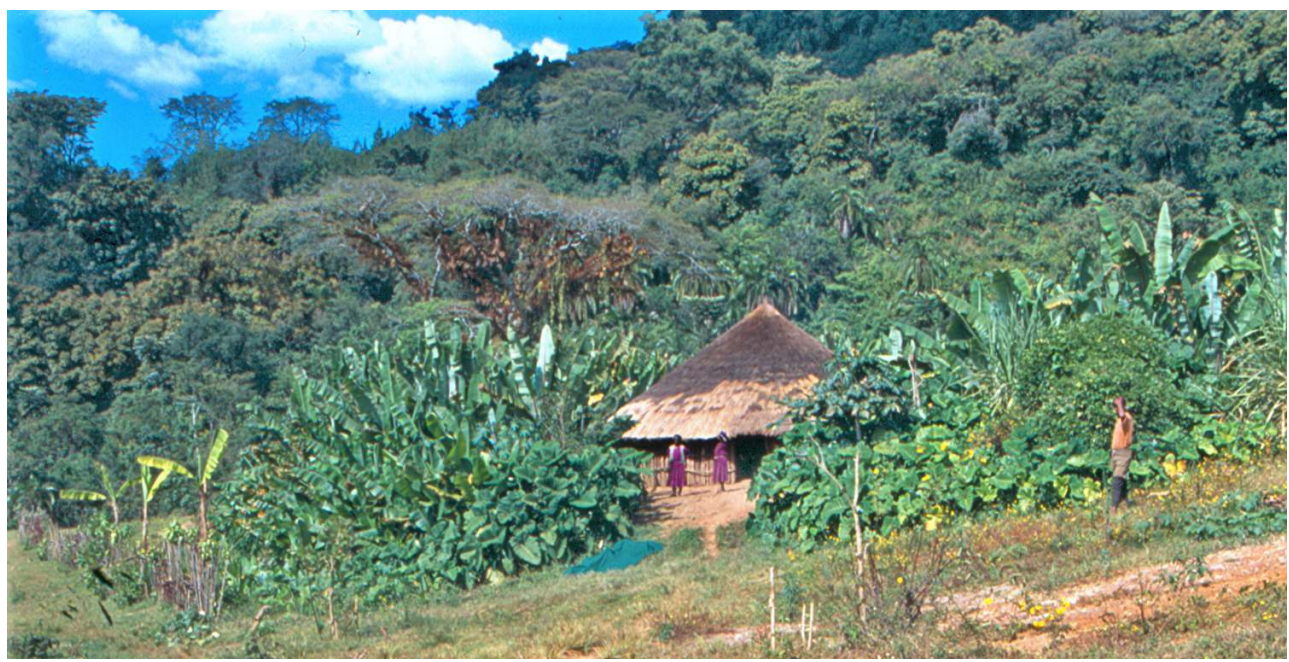

La kororima des forêts aménagées est exploitée ainsi que celle mise en culture dans ces jardins circulaires, autour des maisons

Photo B. R. elles apparaissent souvent disparates et peu coordonnées. S'y impliquent à la fois, des institutions de recherche locales (tel le Bonga Agricultural Research Center), des instances gouvernementales locales (telles les Agricultural and Rural Development Offices), la coopération internationale (Union européenne, Usaid,..) et les ONG telles Farm Africa, SOS Sahel, GTZ... (Brunet \& Boisvert, 2007). Les objectifs affichés concernent certes le développement local mais ils répondent aussi à des préoccupations de valorisation, de gestion durable et de conservation de la biodiversité : la précieuse et menacée Coffee Forest . (Figure 5).

L'Union des coopératives des fermiers de la Coffee Forest (Kafa Coffee Forest Farmers Cooperative Union) est la seule association qui inscrit ouvertement la kororima dans ses objectifs : elle réunit 26 associations de producteurs de diverses localités (Gimbo, Decha, Telo and Gawata) et concerne le café, le miel et les épices: ses actions affichent ouvertement des objectifs internationaux et des préoccupations de protection de la forêt et de meilleures rémunérations des fermiers en recourant à des outils de certification de type «Commerce équitable», «Produits biologiques», "Écocertification ${ }^{13}$ ». Elles agissent à tous les niveaux : amélioration des rendements, domestication de la ressource, augmentation de la qualité, promotion et soutien à la filière, nationale et internationale. Elles imposent donc de nouvelles normes correspondant à des critères (présence de pesticides et insecticides, durabilité sociale et environnementale,..) tous nouveaux pour la filière et décidés en dehors d'elle, souvent sans l'adhésion des acteurs concernés. (Bognetteau et al., 2007) qui s'intéresse notamment aux zones de Bonga ou de Masha, ne sont pas différents: développer une certification sur le miel, le café, le poivre et la kororima en organisant les fermiers et en établissant des liens avec des compagnies privées.

31

Il est manifestement encore bien prématuré de tenter un bilan de toutes ces actions et de vouloir caractériser l'influence de ces projets sur les filières de kororimas. Pour l'instant, 
nos enquêtes ne révèlent guère de changements. On peut cependant remarquer que les provenances issues de la Coffee Forest sont actuellement bien représentées et nombreuses chez les détaillants et chez les grossistes d'Addis-Abeba. Ces derniers signalent une augmentation de l'offre ce qui, pour eux, va plutôt dans le sens d'une baisse des prix, contrariant donc les objectifs affichés d'augmentation de la valeur des productions de la Coffee Forest.

Les marchands d'Addis-Abeba font bien état d'une évolution dans la qualité. Deux points ont retenu leur attention: les fruits sont plus secs et les traces de trous d'enfilage qui constituaient pourtant un signe bien repérable de la spécificité des provenances du Kafa, sont de moins en moins fréquentes. Les problèmes de moisissures et la proportion de fruits pauvres en graines ou vides ont tendance à diminuer, ce que les marchands attribuent à une meilleure maîtrise du moment de cueillette. Ils constatent aussi une amélioration nette de l'homogénéité des fruits ce qui est, peut-être le reflet de la progression de la culture par rapport à la cueillette.

Cependant, la question de la notoriété des provenances ne semble guère avoir évolué et si les détaillants connaissent maintenant plus de noms de provenances, ils ont bien du mal en en donner les spécificités. Quant à la clientèle, elle se contente toujours de se référer aux deux «grandes provenances » Kafa et Gofa. De l'avis des vendeurs, les maîtres mots qui décrivent le mieux l'attitude des consommateurs sont : l'accès facile aux produits, la recherche de la qualité et du moindre coût.

Les urbains ont de plus en plus tendance à privilégier les produits de la kororima qui sont d'usage immédiat et facile à acheter : les ventes en supermarché, dans les boutiques des baltena ou les petits souks de voisinage ont tendance à croître. L'on ne se rend plus au Mercato, sur les qemäma tera, que pour les grandes occasions, par exemple les grandes fêtes religieuses. Les liens traditionnels de confiance entre les détaillants et leurs clients, par où pouvaient passer des conseils concernant la provenance et la qualité, ont tendance à s'amenuiser. Plus question non plus de perdre son temps en décorticage, triage et pilage. Les produits tels que les graines, les poudres et les mélanges tout prêts sont de plus en plus demandés : ces produits transformés sont difficiles à distinguer les uns des autres et si la provenance n'est pas indiquée, il est bien malaisé de les identifier avec certitude.

35 Toutes ces évolutions pourraient peut-être plaider en faveur de la mise en place de signes de qualité et de labels. Pour l'instant, seuls les produits vendus sous les noms des diverses baltena, qui n'ont d'ailleurs pas encore déposé officiellement de marque, sont considérés par les consommateurs comme garantissant un certain niveau de qualité, même si, en ce qui concerne la kororima, nous l'avons vu, il n'existe aucune certitude concernant l'origine des maniguettes employées. Manifestement, les efforts de promotion des labels ou des futures indications géographiques devront intégrer les baltena qui sont maintenant un maillon essentiel dans la commercialisation des épices.

De plus, la qualité telle qu'elle est actuellement perçue au niveau du grand public ne tient pas encore compte des préoccupations de durabilité sociale ou environnementale. À peine observe-t-on, dans la capitale éthiopienne, au niveau des "foires commerciales » et des "ventes caritatives» de plus en plus fréquentes et courues, une tendance, au sein des élites ou d'une clientèle internationale d'expatriés, à privilégier les produits «biologiques" ou "écocertifiés ». Mais ces questions d'impacts environnementaux et sociaux de la production, tant prônés par les projets de développement, ne trouvent encore guère d'échos au niveau de la consommation de masse et les nouvelles normes qui 
y correspondent sont ignorées de la grande majorité des consommateurs mais aussi des acteurs de la filière traditionnelle.

Enfin, un autre obstacle à la réussite de la labellisation des produits issus de la Coffee Forest est lié à la question des prix : le déficit de réputation des provenances de la kororima du Kafa est encore largement responsable du fait que, pour l'instant, elle reste toujours dans une gamme de prix inférieure à celle des maniguettes du Gofa. Le surcoût engendré par la labellisation et les efforts d'amélioration de la qualité n'est pas facilement compensable par une augmentation que le public n'est pas prêt à accepter.

Pour conclure, soulignons à quel point la kororima d'Éthiopie constitue un bon matériel pour interroger une question fondamentale dans le champ de l'étude des productions localisées: les liens qui existent entre les diverses provenances valorisées sur les marchés, la typicité et la qualité de leurs produits (Bérard \& Marchenay, 2004 : p. 77).

Notre étude montre bien qu'en dehors de toute «officialisation » et de (presque) tout contrôle étatique, la qualité des kororimas d'Éthiopie fait l'objet d'un suivi soigneux et d'une évaluation stricte tout le long de la filière : ce sont les acteurs eux-mêmes qui assurent la traçabilité, définissent les critères, se chargent de son contrôle et fixent les prix en conséquence.

D'une manière globale, le niveau de qualité est présenté comme fortement lié à l'origine. La spécificité des provenances n'est pas une préoccupation cardinale pour la clientèle, pauvre ou riche, qui recherche une qualité et des prix liés à ses objectifs et ses possibilités budgétaires. Finalement, les nombreuses provenances éthiopiennes, offrant pourtant tout un éventail de particularités originales, se trouvent rassemblées sur le marché national en deux grandes catégories : l'une, la kororima de Gofa, de qualité supérieure, est plus chère que l'autre, la kororima du Kafa, moins bonne et destinée à des usages courants ou à une clientèle peu fortunée.

41 Cette situation est encore très prégnante et constitue actuellement un obstacle aux projets de promotion des maniguettes issues de la Coffee Forest, à des fins de développement durable local et de conservation de la biodiversité. Malgré des efforts considérables d'amélioration de la qualité, les kororimas issues du Kafa peinent à trouver une clientèle et à atteindre des niveaux de prix satisfaisants : les labellisations et autres signes de qualité dont elles sont bardées n'éveillent pas encore l'intérêt du grand public et les nouvelles normes de qualité basées sur des critères de durabilité passent toujours largement inaperçues. Le succès de ces efforts de valorisation devra probablement s'appuyer sur une opération de séduction et de promotion visant à convaincre d'abord les détaillants et les industriels de la transformation.

\section{BIBLIOGRAPHIE}

Bérard L. \& Marchenay Ph. 2004 - Les produits de terroir, entre cultures et règlements. Paris, CNRS éditions, $229 \mathrm{p}$. 
Bérard L., Cegarra M., Djama M., Louafi L., Marchenay Ph., Roussel B. \& Verdeaux F. (Ed.) 2005 Biodiversité et savoirs naturalistes locaux en France. Cirad, Inra, IFB, Iddri, 271 p.

Bognetteau E., Abebe Haile \& Freerk Wiersum K. 2007 - Linking Forests and People: A potential for sustainable development of the South-West Ethiopian Highlands. Non-Timber Forest Products Research \& Development Project, South-West Ethiopia, 18 p.

Boisvert V., Roussel B. \& K F. (Coord.) 2007 - Pre-selection of Ethiopian Localized Home Garden Products. Report to the Stakeholders Forum. EHGP, Villa Turquoise, Addis Ababa, October 2007, 51 p. + Annexes, 100 p.

Brunet C. \& Boisvert V. 2007 - The Ethiopian spice sector. A case study on kororima (Aframomum corrorima). Ethiopian Home Garden Project. Paris, Addis-Ababa, 66 p.

Chouvin E. 2000 - Catégories populaires et catégories scientifiques. Le cas des oléagineux en Éthiopie. Annales d'Éthiopie, vol. 16, nº $1: 339-360$

Feleke Woldeyes \& Roussel B. 2008 - Ye-Basketo kororima. Ethiopian false-cardamom from Laska. Report on selected Product. Ethiopian Home gardens Project. Paris, Addis-Ababa, MNHN/IRD, EPA, $54 \mathrm{p}$.

Goesttsch E. 1991 - Spice germplasm in Ethiopia. In Engels J.M.M., Hawkes J.G. \& Woredre Melaku. Plant genetetic ressource of Ethiopa. Cambridge University Press : 123-130.

Jansen P.C.M. 1981 - Spices, condiments and medicinal plants in Ethiopia, their taxonomy and agricultural significance. Wageningen, Centre for Agricultural Publishing and Documentation, $327 \mathrm{p}$.

Lock J.M. 1997 - Zingiberaceae. In Edwards S., Sebsebe Demissew \& Hedberg I., Flora of Ethiopia and Eritrea Volume 6. Addis Ababa, Upsala, 324-330.

Roussel B. (Coord.) 2007 - Fact sheets on the studied localised specialities. EHGP component 1. Addis Ababa-Paris, FFEM/EPA, $274 \mathrm{p}$.

Roussel B. \& Verdeaux F. 2007a - Natural patrimony and local communities in Ethiopia : Advantages and limitations of a system of Geographical Indications. In « Nature as local heritage ", Africa, (77) $1: 21-39$

Roussel B. \& Verdeaux F. 2007b - Éthiopie : de l'intérêt des Indications géographiques. In «Biodiversité : savoirs locaux, enjeux global ». $N^{\circ}$ spécial. Courrier de la Planète, 83 : 45-49.

\section{NOTES}

1. Il s'agit d'une coopération franco-éthiopienne financée par le Fonds Français pour l'Environnement Mondial (FFEM) qui met en relation diverses institutions éthiopiennes (EPA, IBCR, EIPO) et françaises (IRD, MNHN, INAO). Le projet s'intitule "Jardins éthiopiens : valorisation des pratiques et des productions et conservation in situ de la biodiversité " : cf. le site www.homegardensofethiopia.com.

2. Faire de la reconnaissance des produits de terroir un instrument de durabilité sociale et environnementale est une idée qui se répand un peu partout dans le monde cf. Bérard et al., (2005) ou encore Bérard \& Marchenay (2005)

3. Outre les recherches conduites par le Projet "Jardins d'Éthiopie", le Programme de recherche Biodivalloc "Des productions localisées aux Indications géographiques : quels instruments pour valoriser la biodiversité dans les pays du Sud ?", financé par l'ANR 2005 (Référence : ANR05 BDIV002), a développé un chantier éthiopien intitulé "Labellisation 
des productions localisées et biodiversité horticole et forestière en Éthiopie" : ce sont les résultats de ces programmes, associant des approches anthropologique, géographique, agronomique et botanique dans l'étude de diverses instruments à l'œuvre en Éthiopie (Commerce équitable, label bio, Indications géographiques,...), qui a fourni le corpus central de données analysées dans cet article.

4. Ce qui signifie littéralement, "savoir-faire domestique". Ces entreprises sont spécialisées dans la confection de préparations culinaires toutes prêtes (poudres de légumineuses, mélanges de farines, etc...) et de condiments. Certaines, tout particulièrement à Addis-Abeba la capitale, se sont bâties de solides réputations commerciales basées sur la qualité et commencent à atteindre des dimensions nationales voire internationales : on peut citer par exemple Salam baltena, Abyssinia baltena, Etsub baltena.

5. Une famille d'une dizaine de personnes prépare trois fois l'an une dizaine de kilogrammes de ce condiment qui contient en moyenne $0,5 \mathrm{~kg}$ de graines de kororima : la consommation annuelle moyenne peut donc être estimée à $1,5 \mathrm{~kg}$ de graines par an.

6. La plante entière est aussi nommée kororima en Oromo, une langue très répandue en Éthiopie, ce qui explique pourquoi Jansen a utilisé le terme pour désigner l'espèce, s'inspirant de la nomenclature choisie par Pereira en 1850 (Jansen 1981). Plusieurs transcriptions du mot kororima se rencontrent dans les documents : kororima, korrorima, korarima (cette dernière étant le plus fréquente dans les textes botaniques cf. Lock 1997). Les autres noms utilisés font référence à des épices aux saveurs proches : poivre d'Abyssinie, fausse cardamome, fausse muscade, etc. La kororima est très proche de la "graine de paradis", célèbre épice, connue en Europe dès le Moyen Age sous le nom de maniguette ou de malaguette, issue de diverses espèces d'Aframomum d'Afrique centrale et occidentale.

7. Si la plupart des détaillants se limitent ainsi à ces deux appellations, certains, en vrais spécialistes, distinguent plus de provenances et offrent plus de choix à leur clientèle. C'est d'ailleurs, nous y reviendrons, une tendance actuelle.

8. Il est difficile de donner avec précision la proportion relative sur les marchés d'Addis-Abeba, de ces deux provenances : les kororimas de Gofa sont un peu moins fréquentes : $40 \%$ pourrait être une estimation plausible mais la promotion dont bénéficient actuellement les kororimas du Kafa oblige à revoir ce chiffre à la baisse.

9. Les paysans utilisent le terme amharique zuriya (que l'on peut traduire par "famille") : la rotondité du fruit n'est pas liée à la "famille" de la plante.

10. Il ne faut pas cependant faire de ce ces deux traits un critère absolu : certaines maniguettes du Gofa sont aussi séchées ainsi ; de plus en plus dans le but d'améliorer la qualité, certaines kororimas du Kafa sont aussi séchées au soleil et non percées.

11. Il existe une autorité fédérale chargée de ces questions : Qsae : Quality and Standard Authority of Ethiopia. Elle s'est dotée en 2001 d'une norme de qualité concernant la commercialisation des fruits et graines de kororima ("Ethiopian cardamom (korarima) capsules and seeds") : ref. FDES 684 : elle insiste surtout sur l'état de séchage des produits, la présence de moisissures, l'intégrité des fruits et l'arôme des grains, tous critères retenus aussi par les divers agents de la filière.

12. 1 euro équivaut actuellement à environ 15 birrs (avril 2009).

13. Par exemple, les labels et signes "Max Havelaar", "Rain Forest Alliance" ou "AB" sont présents dans le Kafa. 


\section{RÉSUMÉS}

Les graines de la fausse cardamome kororima, Aframomum corrorima (Braun) Jansen, maniguette endémique d'Éthiopie, constituent une épice indispensable, emblématique du pays tout entier, aux usages multiples et quasi quotidiens. Plante spontanée des sous-bois des forêts humides du Kafa, c'est aussi une plante cultivée dans les jardins de diverses régions de l'Éthiopie méridionale.

Cet article, à partir de l'analyse de la production et des filières de commercialisation de la kororima, se propose de mettre en lumière les relations qui lient provenances, spécificité et qualité des produits. Sur le marché national, les diverses productions localisées d'Éthiopie finissent par nourrir deux filières bien disjointes dont les produits finaux sont désignés du nom de deux zones principales de production : classiquement, la kororima du Gofa et la kororima du Kafa ne se concurrencent pas et sont toutes les deux présentes dans les boutiques et sur les étalages d'épices des marchés d'Addis-Abeba. Parfaitement distinguées par tous les acteurs de la filière qui connaissent leurs spécificités (aspects, couleur, odeur, goût), elles sont considérées comme deux produits de qualité différente, reflets de conditions de production (spontanée/ cultivée) et de modes de préparation et de séchage. Elles sont destinées à deux types de clientèles et d'usages. La kororima de Gofa, de qualité supérieure, est vendue plus cher, à une clientèle plus aisée et pour des usages plus valorisés.

Depuis une petite décennie, les efforts de valorisation des produits issus de l'écosystème coffee forest et des jardins d'Éthiopie, pour conserver la biodiversité forestière et horticole, ont vu dans la kororima un produit idéal: plusieurs tentatives de développement, de promotion et de commercialisation ont eu lieu. Celles qui concernent la kororima du Kafa, dont la qualité a été améliorée, se heurtent aux habitudes de consommation et de commercialisation: les provenances nouvellement valorisées ont du mal à faire reconnaître leur spécificité et à se tailler une place sur les marchés.

Seeds of false cardamom kororima (Aframomum corrorima (Braun) Jansen), malagueta pepper endemic of Ethiopia, is an unavoidable spice, emblematic of the whole country with multiple uses and quite routine uses. Spontaneous within the lowest stratum of the Kafa moist forest, it is also a cultivated plant in the gardens of several regions in southern Ethiopia.

This paper, starting from the analysis of production and commercial chains of kororima, aims to put light on the relation between provenances, specificity and quality on the national Ethiopian market. At the national level, various localized productions of kororima converge into two main commercial chains well separated of which final products are named after the two main zones of production : classically, kororima from Gofa and kororima from Kafa are not competing and one can find the two provenances in each shop and spice corners of Addis Ababa markets. Clearly distinguished by the actors of the commercial chain who recognize perfectly the specificities (aspect, colour, odour, taste), they are considered as two products of two different qualities resulting from conditions of production (spontaneous/cultivated), way of transformation, and drying processes. 
Since a few decades, there have been valorisation efforts of products coming from coffee forest ecosystems as well as homegardens of Ethiopia in order to contribute to conservation of the rich and unique forest and garden biodiversity. In this context, kororima appears as a very convenient product and numerous initiatives of commercialization have been set up.

These two products are targeted/end up for two categories of consumers and utilization. Gofa kororima, which is of higher quality, is sold at higher price to well off consumer for more valorised purposes. Recent effort of promoting Kafa kororima, which quality has been ameliorated, is facing to the consumption habit and existing commercialization patterns. Various provenances which quality has been ameliorated have some difficulties to find a good place in the market.

INDEX

Mots-clés : Aframomum corrorima, commerce, appellation d'origine

Keywords : Aframomum corrorima, Ethiopia

Index géographique : Éthiopie

\section{AUTEURS}

BERNARD ROUSSEL

MNHN/IRD, UMR « Patrimoines Locaux », Paris

\section{FELEKE WOLDEYES}

Université d'Arba Minch, Éthiopie 